\title{
The Annual Modulation Signature for Dark Matter: DAMA/LIBRA-Phase 1 Results and Perspectives
}

\author{
Rita Bernabei, ${ }^{1,2}$ Pierluigi Belli, ${ }^{2}$ Fabio Cappella, ${ }^{3,4}$ Vincenzo Caracciolo, ${ }^{5}$ \\ Simone Castellano, ${ }^{5}$ Riccardo Cerulli, ${ }^{5}$ Chang Jang Dai, ${ }^{6}$ Annelisa d'Angelo, ${ }^{3,4}$ \\ Silio d'Angelo, ${ }^{1,2}$ Alessandro Di Marco, ${ }^{1,2}$ H. L. He, ${ }^{6}$ Antonella Incicchitti, ${ }^{4}$ H. H. Kuang, ${ }^{6}$ \\ X. H. Ma, ${ }^{6}$ Francesco Montecchia, ${ }^{2,7}$ X. D. Sheng, ${ }^{6}$ Rui Guang Wang, ${ }^{6}$ and Zi-Piao Ye ${ }^{6,8}$ \\ ${ }^{1}$ Dipartimento di Fisica, Università di Roma "Tor Vergata", 00133 Rome, Italy \\ ${ }^{2}$ INFN, sez. Roma “Tor Vergata”, 00133 Rome, Italy \\ ${ }^{3}$ Dipartimento di Fisica, Università di Roma "La Sapienza", 00185 Rome, Italy \\ ${ }^{4}$ INFN, sez. Roma, 00185 Rome, Italy \\ ${ }^{5}$ Laboratori Nazionali del Gran Sasso, INFN, 67100 Assergi, Italy \\ ${ }^{6}$ Institute of High Energy Physics, Chinese Academy of Sciences, P.O. Box 918-3, Beijing 100049, China \\ ${ }^{7}$ Dipartimento di Ingegneria Civile e Ingegneria Informatica, Università di Roma "Tor Vergata", 00133 Rome, Italy \\ ${ }^{8}$ Maths \& Physics College, Jinggangshan University, Jián 343009, China
}

Correspondence should be addressed to Pierluigi Belli; pierluigi.belli@roma2.infn.it

Received 13 January 2014; Accepted 19 March 2014; Published 24 June 2014

Academic Editor: Anselmo Meregaglia

Copyright (C) 2014 Rita Bernabei et al. This is an open access article distributed under the Creative Commons Attribution License, which permits unrestricted use, distribution, and reproduction in any medium, provided the original work is properly cited. The publication of this article was funded by $\mathrm{SCOAP}^{3}$.

\begin{abstract}
The results obtained with the total exposure of 1.04 ton $\times$ yr collected by DAMA/LIBRA-phasel deep underground at the Gran Sasso National Laboratory (LNGS) of the I.N.F.N. during 7 annual cycles are summarized. The DAMA/LIBRA-phasel and the former DAMA/NaI data (cumulative exposure 1.33 ton $\times$ yr, corresponding to 14 annual cycles) give evidence at $9.3 \sigma$ C.L. for the presence of Dark Matter (DM) particles in the galactic halo, on the basis of the exploited model independent DM annual modulation signature by using highly radiopure $\mathrm{NaI}(\mathrm{Tl})$ target. The modulation amplitude of the single-hit events in the (2-6) keV energy interval is $(0.0112 \pm 0.0012) \mathrm{cpd} / \mathrm{kg} / \mathrm{keV}$; the measured phase is $(144 \pm 7)$ days and the measured period is $(0.998 \pm 0.002) \mathrm{yr}$; values are in a good well in agreement with those expected for DM particles. No systematic or side reactions able to mimic the exploited DM signature have been found or suggested by anyone over more than a decade. Some of the perspectives of the presently running DAMA/LIBRA-phase2 are outlined.
\end{abstract}

\section{Introduction}

The presently running DAMA/LIBRA [1-11] experiment, as the former DAMA/NaI [12-41], has the main aim to investigate the presence of DM particles in the galactic halo by exploiting the model independent DM annual modulation signature (originally suggested in $[42,43]$ ). Moreover, the developed highly radiopure $\mathrm{NaI}(\mathrm{Tl})$ target-detectors [1] assure as well sensitivity to a wide range of DM candidates, interaction types, and astrophysical scenarios.
As a consequence of the Earth's revolution around the Sun, the Earth should be crossed by a larger flux of DM particles around $\simeq 2$ June and by a smaller one around $\simeq 2$ December. This DM annual modulation signature is very distinctive since the effect induced by DM particles must simultaneously satisfy all the following requirements: (1) the rate must contain a component modulated according to a cosine function (2) with one year period and (3) a phase that peaks roughly $\simeq 2$ June; (4) this modulation must only be found in a well-defined low energy range, where DM particle 
induced events can be present; (5) it must apply only to those events in which just one detector of many actually "fires" (single-hit events), since the DM particle multi-interaction probability is negligible; (6) the modulation amplitude in the region of maximal sensitivity must be $\simeq 7 \%$ for usually adopted halo distributions (see, e.g., $[8,23,24]$ ), but it can be larger in case of some possible scenarios such as, for example, those in [44-48] (even up to $\simeq 30 \%$ ). Thus, this signature is model-independent, is very effective, and, in addition, it allows the test of a large range of cross sections and of halo densities.

This DM signature might be mimicked only by systematic effects or side reactions able to account for the whole observed modulation amplitude and to simultaneously satisfy all the requirements given above. No one is available [1$3,7,8,22-24]$.

The full description of the DAMA/LIBRA setup during phasel and other related arguments have been discussed in detail in $[1-4,8]$ and references therein. Here, we just remind the reader that the sensitive part of this setup is made of 25 highly radiopure $\mathrm{NaI}(\mathrm{Tl})$ crystal scintillators (5 rows by 5-column matrix) having $9.70 \mathrm{~kg}$ mass each one. In each detector two $10 \mathrm{~cm}$ long UV light guides (made of Suprasil B quartz) act also as optical windows on the two end faces of the crystal and are coupled with two low background photomultipliers (PMTs) working in coincidence at single photoelectron level. The low background 9265-B53/FL and 9302-A/FL PMTs, developed by EMI-Electron Tubes with dedicated R\&Ds, were used in the phasel; for details, see $[1,21,23]$ and references therein. The detectors are housed in a sealed low-radioactive copper box installed in the center of a low-radioactive $\mathrm{Cu} / \mathrm{Pb} / \mathrm{Cd}$-foils/polyethylene/paraffin shield; moreover, about $1 \mathrm{~m}$ concrete (made from the Gran Sasso rock material) almost fully surrounds (mostly outside the barrack) this passive shield, acting as a further neutron moderator. A threefold-level sealing system prevents the detectors from being in contact with the environmental air of the underground laboratory [1]. The light response of the detectors during phasel typically ranges from 5.5 to 7.5 photoelectrons $/ \mathrm{keV}$, depending on the detector. The hardware threshold of each PMT is at single photoelectron, while a software energy threshold of $2 \mathrm{keV}$ electron equivalent (hereafter keV) is used [1, 21]. Energy calibration with Xrays $/ \gamma$ sources are regularly carried out in the same running condition down to few $\mathrm{keV}$ [1]; in particular, double coincidences due to internal X-rays from ${ }^{40} \mathrm{~K}$ (which is at ppt levels in the crystals) provide (when summing the data over long periods) a calibration point at $3.2 \mathrm{keV}$ close to the software energy threshold (for details, see [1]). The radiopurity, the procedures, and details are discussed in [1$4,8]$ and references therein.

\section{The Results of DAMA/LIBRA-Phase1 and DAMA/NaI}

The total exposure of DAMA/LIBRA-phasel is 1.04 ton $\times$ yr in seven annual cycles; when including also that of the first generation DAMA/NaI experiment, it is 1.33 ton $\times \mathrm{yr}$,

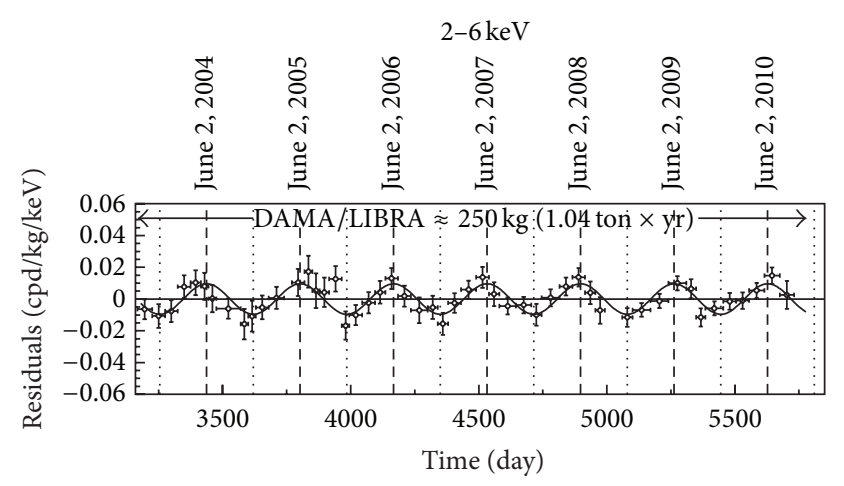

FIGURE 1: Experimental residual rate of the single-hit scintillation events measured by DAMA/LIBRA-phasel in the (2-6) keV energy interval as a function of the time. The data points present the experimental errors as vertical bars and the associated time bin width as horizontal bars. The superimposed curves are the cosinusoidal functions behaviour $\mathrm{A} \cos \omega\left(t-t_{0}\right)$ with a period $T=2 \pi / \omega=1 \mathrm{yr}$, a phase $t_{0}=152.5$ day (June 2nd) and modulation amplitudes, $A$, equal to the central values obtained by best fit on the data points of the entire DAMA/LIBRA-phasel. The dashed vertical lines correspond to the maximum expected for the DM signal (June 2nd), while the dotted vertical lines correspond to the minimum.

corresponding to 14 annual cycles. The variance of the cosine $\left(\alpha-\beta^{2}\right)=\left(\left\langle\cos ^{2}\right\rangle-\langle\cos \rangle^{2}\right)$, where the averages $\langle\cdots\rangle$ are taken over the periods in which the data taking was on, is 0.518 during the DAMA/LIBRA-phasel, showing that the setup has been operational evenly throughout the years (the expectation value for full-year data taking is $\left.\left(\alpha-\beta^{2}\right)=0.5\right)$. For details, see $[2-4,8]$.

The total number of events collected for the routine calibrations during the entire DAMA/LIBRA-phasel is about $9.6 \times 10^{7}$, while about $3.5 \times 10^{6}$ events $/ \mathrm{keV}$ have been collected for the evaluation of the acceptance window efficiency for noise rejection near energy threshold [1]. The duty cycle of the experiment is high [4]; the routine calibrations and, in particular, those related to the acceptance windows efficiency mainly affect it.

Figure 1 shows the time behaviour of the experimental residual rates of the single-hit scintillation events in the (2-6) keV energy interval for DAMA/LIBRA-phasel. The residuals of the DAMA/NaI data $(0.29$ ton $\times$ yr) are given in $[2,8,23,24]$. We remind the reader that these residual rates are calculated from the measured rate of the single-hit events after subtracting the constant part: $\left\langle r_{i j k}-\text { flat }_{j k}\right\rangle_{j k}$. Here, $r_{i j k}$ is the rate in the considered $i$ th time interval for the $j$ th detector in the $k$ th energy bin, while flat ${ }_{j k}$ is the rate of the $j$ th detector in the $k$ th energy bin averaged over the cycles; it is of order of $\leqslant 1 \mathrm{cpd} / \mathrm{kg} / \mathrm{keV}[1,2,49]$. The average is made on all the detectors ( $j$ index) and on all the energy bins ( $k$ index) which constitute the considered energy interval. The weighted mean of the residuals must obviously be zero over one cycle.

The $\chi^{2}$ test excludes the hypothesis of absence of modulation in the data: $\chi^{2} /$ d.o.f. $=83.1 / 50$ and the $P$ value is $P=2.2 \times 10^{-3}$ for the (2-6) keV energy interval. When fitting 
the single-hit residual rate of DAMA/LIBRA-phasel (Figure 1) together with the DAMA/NaI ones, with the function, A $\cos \omega\left(t-t_{0}\right)$, considering a period $T=2 \pi / \omega=1 \mathrm{yr}$ and a phase $t_{0}=152.5$ day (June 2nd) as expected by the $\mathrm{DM}$ annual modulation signature, the following modulation amplitude is obtained: $A=(0.0110 \pm 0.0012) \mathrm{cpd} / \mathrm{kg} / \mathrm{keV}$ corresponding to $9.2 \sigma$ C.L. ( $\chi^{2}$ of the fit is 70.4 over 86 d.o.f.).

When the period and the phase are kept free in the fitting procedure, the modulation amplitude is $(0.0112 \pm$ $0.0012) \mathrm{cpd} / \mathrm{kg} / \mathrm{keV}$ (9.3 $\sigma$ C.L.), the period $T=(0.998 \pm$ $0.002)$ year, and the phase $t_{0}=(144 \pm 7)$ day. The period and the phase are well compatible with expectations for a DM annual modulation signal. In particular, the phase is consistent with about June 2 nd and is fully consistent with the value independently determined by Maximum Likelihood analysis (see later). For completeness, we recall that a slight energy dependence of the phase could be expected in case of possible contributions of nonthermalized DM components to the galactic halo, such as, for example, the SagDEG stream [26, 50-54] and the caustics [55]. For more details, see [4].

The modulation amplitudes singularly calculated for each annual cycle of DAMA/NaI and DAMA/LIBRA-phasel are compatible among them and are normally fluctuating around their best fit values [2-4]. In particular, for the (2-6) keV energy interval, the $\chi^{2}$ is 10.8 over 13 d.o.f. corresponding to an upper tail probability of $63 \%$, while the run test yields a lower tail probability of $23 \%$. This analysis confirms that the data collected in all the annual cycles with DAMA/NaI and DAMA/LIBRA-phasel are statistically compatible and can be considered together, on the contrary of the statements in [56].

The DAMA/LIBRA-phasel single-hit residuals of Figure 1 and those of DAMA/NaI have also been investigated by a Fourier analysis. The data analysis procedure has been described in detail in [8]. A clear peak corresponding to a period of 1 year (see Figure 2) is evident for the (2-6) keV energy interval; the same analysis in the (6-14) keV energy region shows instead only aliasing peaks. No other structure at different frequencies has been observed (see also [8]).

Absence of any other significant background modulation in the energy spectrum has been verified in energy regions not of interest for DM. (In fact, the background in the lowest energy region is essentially due to "Compton" electrons, $\mathrm{X}$ rays, and/or Auger electrons, muon induced events, etc., which are strictly correlated with the events in the higher energy region of the spectrum. Thus, if a modulation detected in the lowest energy region was due to a modulation of the background (rather than to a signal), an equal or larger modulation in the higher energy regions should be present.) For example, the measured rate integrated above $90 \mathrm{keV}, R_{90}$, as a function of the time has been analysed [4]. Similar result is obtained when comparing the single-hit residuals in the (2-6) $\mathrm{keV}$ with those in other energy intervals; for example, Figure 3 shows the single-hit residuals in the (2-6) $\mathrm{keV}$ and in the (6-14) keV energy regions for the entire DAMA/LIBRAphasel data as if they were collected in a single annual cycle (i.e., binning in the variable time from the Jan 1st of each annual cycle). It is worth noting that the obtained results account for whatever kind of background and, in

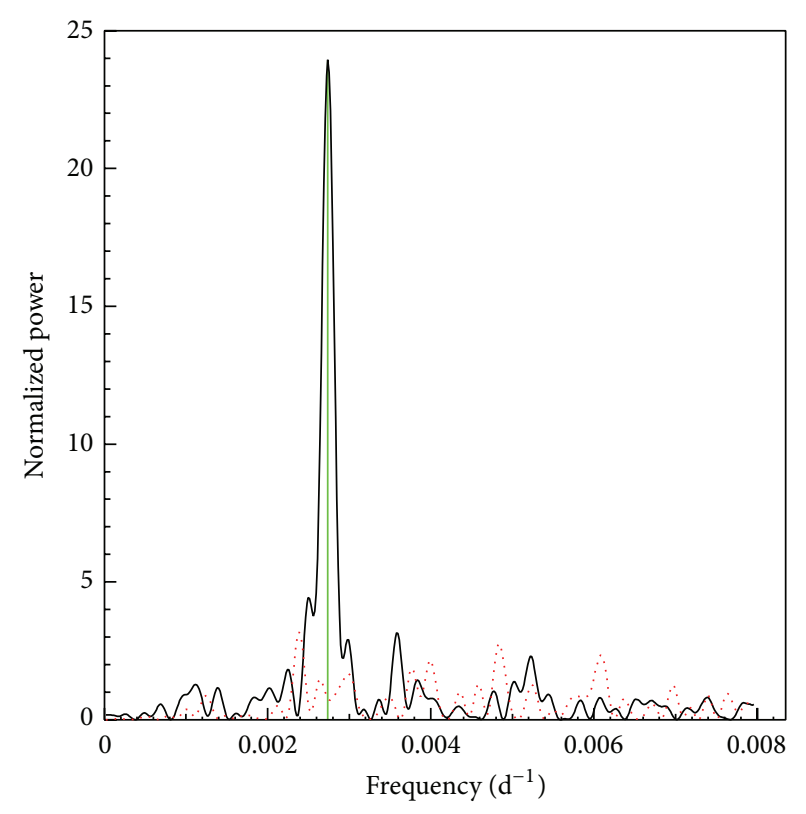

FIGURE 2: Power spectrum of the measured single-hit residuals in the (2-6) keV (solid lines) and (6-14) keV (dotted lines) energy intervals calculated according to [8], including also-as usual in DAMA analyses-the treatment of the experimental errors and of the time binning. The data refer to DAMA/NaI and DAMA/LIBRAphasel. As it can be seen, the principal mode present in the (26) $\mathrm{keV}$ energy interval corresponds to a frequency of $2.737 \times 10^{-3} \mathrm{~d}^{-1}$ (vertical lines), corresponding to a period of $\simeq 1$ year. A similar peak is not present in the (6-14) keV energy interval.

addition, no background process able to mimic the DM annual modulation signature (that is able to simultaneously satisfy all the peculiarities of the signature and to account for the measured modulation amplitude) is available (see also discussions, for example, in [1-4, 7, 8, 49, 57-62]).

A further relevant investigation in the DAMA/LIBRAphasel data has been performed by applying the same hardware and software procedures, used to acquire and to analyse the single-hit residual rate, to the multiple-hit one. (A multiple-hit event is defined when more scintillating pulses arrive within a time window of about $600 \mathrm{~ns}$.) In fact, since the probability that a DM particle interacts in more than one detector is negligible, a DM signal can be present just in the single-hit residual rate. Thus, the comparison of the results of the single-hit events with those of the multiple-hit ones corresponds practically to compare between them the cases of DM particles beam-on and beam-off. This procedure also allows an additional test of the background behaviour in the same energy interval where the positive effect is observed. In particular, in Figure 4, the residual rates of the singlehit events measured over the DAMA/LIBRA-phasel annual cycles are reported, as collected in a single cycle, together with the residual rates of the multiple-hit events, in the (26) $\mathrm{keV}$ energy interval. While, as already observed, a clear modulation, satisfying all the peculiarities of the DM annual modulation signature, is present in the single-hit events, the fitted modulation amplitude for the multiple-hit residual rate is well compatible with zero: $-(0.0005 \pm 0.0004) \mathrm{cpd} / \mathrm{kg} / \mathrm{keV}$ 


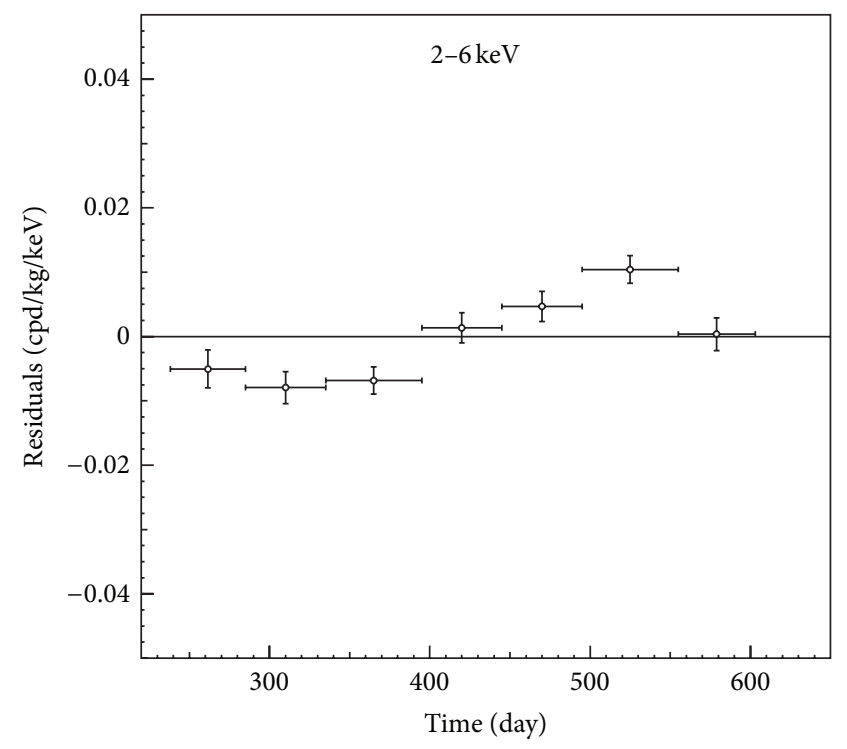

(a)

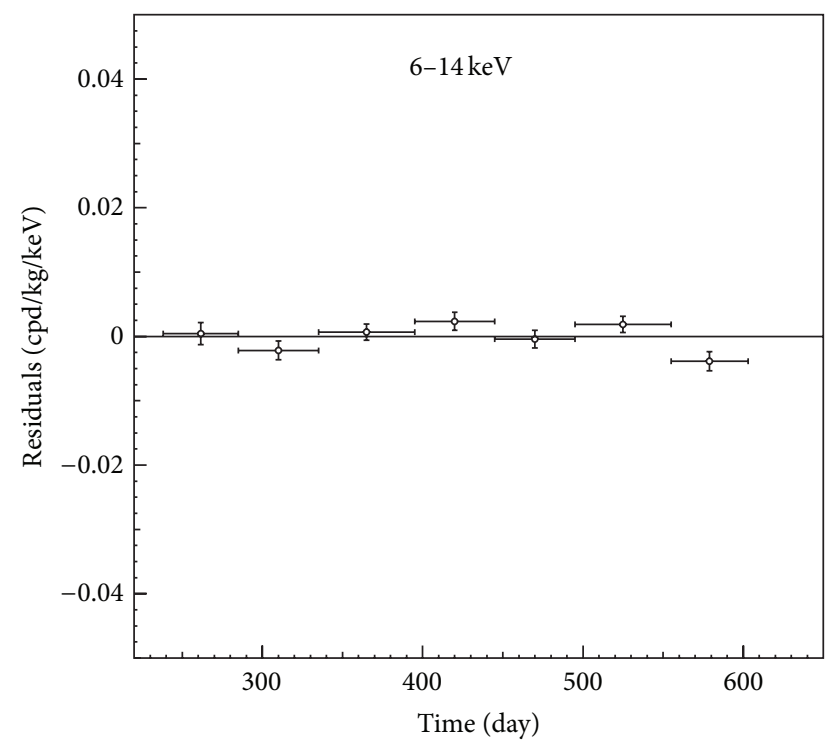

(b)

FIGURE 3: Experimental single-hit residuals in the (2-6) keV and in the (6-14) keV energy regions for the entire DAMA/LIBRA-phasel data as if they were collected in a single annual cycle (i.e., binning in the variable time from the Jan 1st of each annual cycle). The data points present the experimental errors as vertical bars and the associated time bin width as horizontal bars. The initial time of the figures is taken at August 7th. A clear modulation satisfying all the peculiarities of the DM annual modulation signature is present in the lowest energy interval with $A=(0.0088 \pm 0.0013) \mathrm{cpd} / \mathrm{kg} / \mathrm{keV}$, while it is absent just above $A=(0.00032 \pm 0.00076) \mathrm{cpd} / \mathrm{kg} / \mathrm{keV}$.

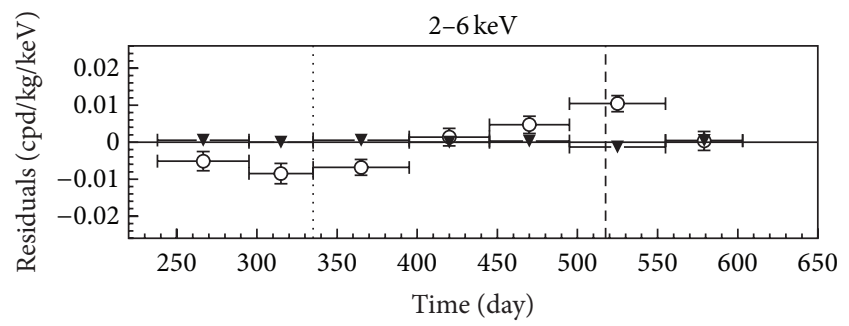

FIGURE 4: Experimental residual rates of DAMA/LIBRA-phasel single-hit events (open circles), class of events to which DM events belong, and, for multiple-hit events (filled triangles), class of events to which DM events do not belong. They have been obtained by considering for each class of events the data as collected in a single annual cycle and by using in both cases the same identical hardware and the same identical software procedures. The initial time of the figure is taken on August 7th. The experimental points present the errors as vertical bars and the associated time bin width as horizontal bars. Analogous results were obtained for the DAMA/NaI data [24].

in the energy region (2-6) $\mathrm{keV}$. Thus, again evidence of annual modulation with proper features as required by the DM annual modulation signature is present in the single-hit residuals (events class to which the DM particle induced events belong), while it is absent in the multiple-hit residual rate (event class to which only background events belong). Similar results were also obtained for the last two annual cycles of the DAMA/NaI experiment [24]. Since the same identical hardware and the same identical software procedures have been used to analyse the two classes of events, the obtained result offers an additional strong support for the presence of a DM particle component in the galactic halo.

The annual modulation present at low energy can also be pointed out by depicting - as a function of the energythe modulation amplitude, $S_{m, k}$, obtained by maximum likelihood method over the data considering $T=1 \mathrm{yr}$ and $t_{0}=$ 152.5 day. For such purpose, the likelihood function of the single-hit experimental data in the $k$ th energy bin is defined as $\mathbf{L}_{\mathbf{k}}=\Pi_{i j} e^{-\mu_{i j k}}\left(\mu_{i j k}^{N_{i j k}} / N_{i j k} !\right)$, where $N_{i j k}$ is the number of events collected in the $i$ th time interval (hereafter 1 day), by the $j$ th detector and in the $k$ th energy bin. $N_{i j k}$ follows a Poisson's distribution with expectation value $\mu_{i j k}=\left[b_{j k}+\right.$ $\left.S_{i k}\right] M_{j} \Delta t_{i} \Delta E \epsilon_{j k}$. The $b_{j k}$ are the background contributions, $M_{j}$ is the mass of the $j$ th detector, $\Delta t_{i}$ is the detector running time during the $i$ th time interval, $\Delta E$ is the chosen energy bin, $\epsilon_{j k}$ is the overall efficiency (for details, see, e.g., [1]). Moreover, the signal can be written as $S_{i k}=S_{0, k}+S_{m, k} \cdot \cos \omega\left(t_{i}-t_{0}\right)$, where $S_{0, k}$ is the constant part of the signal and $S_{m, k}$ is the modulation amplitude. The usual procedure is to minimize the function $y_{k}=-2 \ln \left(\mathbf{L}_{\mathbf{k}}\right)$ - const for each energy bin; the free parameters of the fit are the $\left(b_{j k}+S_{0, k}\right)$ contributions and the $S_{m, k}$ parameter. Hereafter, the index $k$ is omitted for simplicity.

In Figure 5, the obtained $S_{m}$ are shown in each considered energy bin (there $\Delta E=0.5 \mathrm{keV}$ ) when the data of DAMA/NaI and DAMA/LIBRA-phasel are considered. It can be inferred that positive signal is present in the (2-6) keV energy interval, while $S_{m}$ values compatible with zero are present just above. In fact, the $S_{m}$ values in the (6-20) keV energy interval have random fluctuations around zero with $\chi^{2}$ equal to 35.8 for 28 
TABLE 1: Best fit values for the (2-6) and (6-14) keV energy intervals ( $1 \sigma$ errors) for $S_{m}$ versus $Z_{m}$ and $Y_{m}$ versus $t^{*}$, considering the cumulative exposure of DAMA/NaI and DAMA/LIBRA-phasel. See also Figure 6.

\begin{tabular}{llccc}
\hline$E(\mathrm{keV})$ & $S_{m}(\mathrm{cpd} / \mathrm{kg} / \mathrm{keV})$ & $Z_{m}(\mathrm{cpd} / \mathrm{kg} / \mathrm{keV})$ & $Y_{m}(\mathrm{cpd} / \mathrm{kg} / \mathrm{keV})$ & $t^{*}(\mathrm{day})$ \\
\hline $2-6$ & $(0.0106 \pm 0.0012)$ & $-(0.0006 \pm 0.0012)$ & $(0.0107 \pm 0.0012)$ & $(149.5 \pm 7.0)$ \\
$6-14$ & $(0.0001 \pm 0.0007)$ & $(0.0000 \pm 0.0005)$ & $(0.0001 \pm 0.0008)$ & Undefined \\
\hline
\end{tabular}

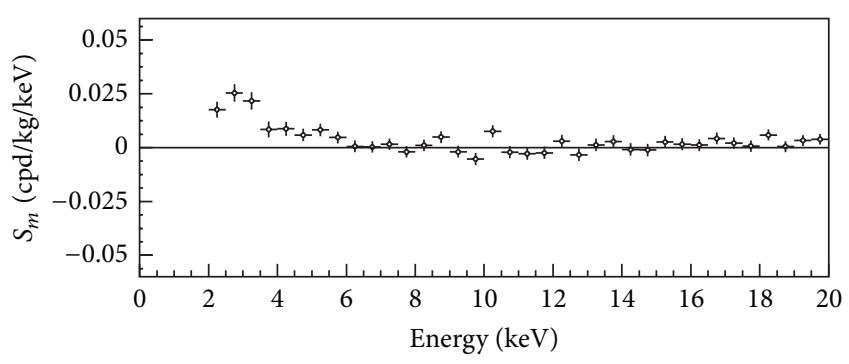

Figure 5: $S_{m}$ variable as a function of the energy for the total cumulative exposure 1.33 ton $\times$ yr. The energy bin is $0.5 \mathrm{keV}$. A clear modulation is present in the lowest energy region, while $S_{m}$ values compatible with zero are present just above. In fact, the $S_{m}$ values in the (6-20) keV energy interval have random fluctuations around zero with $\chi^{2}$ equal to 35.8 for 28 degrees of freedom (upper tail probability of $15 \%)$.

degrees of freedom (upper tail probability of 15\%). All this confirms the previous analyses.

As described in $[2-4,8]$, the observed annual modulation effect is well distributed in all the 25 detectors at 95\% C.L.

Among further additional tests, the analysis of the modulation amplitudes as a function of the energy separately for the nine inner detectors and the remaining external ones has been carried out for the entire DAMA/LIBRA-phasel. The obtained values are fully in agreement; in fact, the hypothesis that the two sets of modulation amplitudes as a function of the energy belong to the same distribution has been verified by $\chi^{2}$ test, obtaining $\chi^{2} /$ d.o.f. $=3.9 / 4$ and $8.9 / 8$ for the energy intervals $(2-4)$ and $(2-6) \mathrm{keV}$, respectively $(\Delta E=0.5 \mathrm{keV})$. This shows that the effect is also well shared between inner and outer detectors.

Let us, finally, release the assumption of a phase $t_{0}=152.5$ day in the procedure to evaluate the modulation amplitudes. In this case, the signal can be written as

$$
\begin{aligned}
S_{i k} & =S_{0, k}+S_{m, k} \cos \omega\left(t_{i}-t_{0}\right)+Z_{m, k} \sin \omega\left(t_{i}-t_{0}\right) \\
& =S_{0, k}+Y_{m, k} \cos \omega\left(t_{i}-t^{*}\right) .
\end{aligned}
$$

For signals induced by DM particles, one should expect the following: (i) $Z_{m, k} \sim 0$ (because of the orthogonality between the cosine and the sine functions); (ii) $S_{m, k} \simeq Y_{m, k}$; (iii) $t^{*} \simeq t_{0}=152.5$ day. In fact, these conditions hold for most of the dark halo models; however, as mentioned above, slight differences can be expected in case of possible contributions from nonthermalized DM components, such as, for example, the SagDEG stream [26, 50-54] and the caustics [55].

Considering cumulatively the data of DAMA/NaI and DAMA/LIBRA-phasel (exposure 1.33 ton $\times$ yr) the obtained
$2 \sigma$ contours in the plane $\left(S_{m}, Z_{m}\right)$ for the (2-6) keV and (614) $\mathrm{keV}$ energy intervals are shown in Figure 6(a), while in Figure $6(\mathrm{~b})$ the obtained $2 \sigma$ contours in the plane $\left(Y_{m}, t^{*}\right)$ are depicted. The best fit values for the (2-6) and (6-14) keV energy intervals ( $1 \sigma$ errors) for $S_{m}$ versus $Z_{m}$ and $Y_{m}$ versus $t^{*}$ are reported in Table 1 .

Finally, setting $S_{m}$ in (1) to zero, the $Z_{m}$ values as function of the energy have also been determined by using the same procedure. The values of $Z_{m}$ as a function of the energy is reported in Figure 7; they are expected to be zero. The $\chi^{2}$ test applied to the data supports the hypothesis that the $Z_{m}$ values are simply fluctuating around zero; in fact, for example, in the (2-14) $\mathrm{keV}$ and (2-20) $\mathrm{keV}$ energy region the $\chi^{2} /$ d.o.f. are equal to $23.0 / 24$ and $46.5 / 36$ (probability of $52 \%$ and $11 \%$ ), respectively.

The behaviour of the phase $t^{*}$ variable as function of energy is shown in Figure 8 for the cumulative exposure of DAMA/NaI and DAMA/LIBRA-phasel (1.33 ton $\times$ yr). No modulation is present above $6 \mathrm{keV}$ and the phase is undetermined.

Sometimes naive statements were put forward as the fact that, in nature, several phenomena may show some kind of periodicity. It is worth noting that the point is whether they might mimic the annual modulation signature in DAMA/LIBRA (and former DAMA/NaI), that is, whether they might be not only quantitatively able to account for the observed modulation amplitude but also able to contemporaneously satisfy all the requirements of the DM annual modulation signature. The same is also for side reactions. This has already been deeply investigated in [1-4] and references therein; the arguments and the quantitative conclusions, presented there, also apply to the entire DAMA/LIBRAphasel data. Additional arguments can be found in $[7,8,49$, 57-62].

Firstly, in order to continuously monitor the running conditions, several pieces of information are acquired with the production data and quantitatively analysed. In particular, all the time behaviour of the running parameters, acquired with the production data, have been investigated. Table 2 shows the modulation amplitudes obtained for each annual cycle when fitting the time behaviour of the values of the main parameters including a cosine modulation with the same phase and period as for DM particles. As can be seen, all the measured amplitudes are well compatible with zero.

No modulation has been found in any possible source of systematics or side reactions; thus, cautious upper limits $(90 \%$ C.L.) on possible contributions to the DAMA/LIBRA measured modulation amplitude are summarized in Table 3 (also see [2-4]). It is worth noting that they do not quantitatively account for the measured modulation amplitudes and also are 


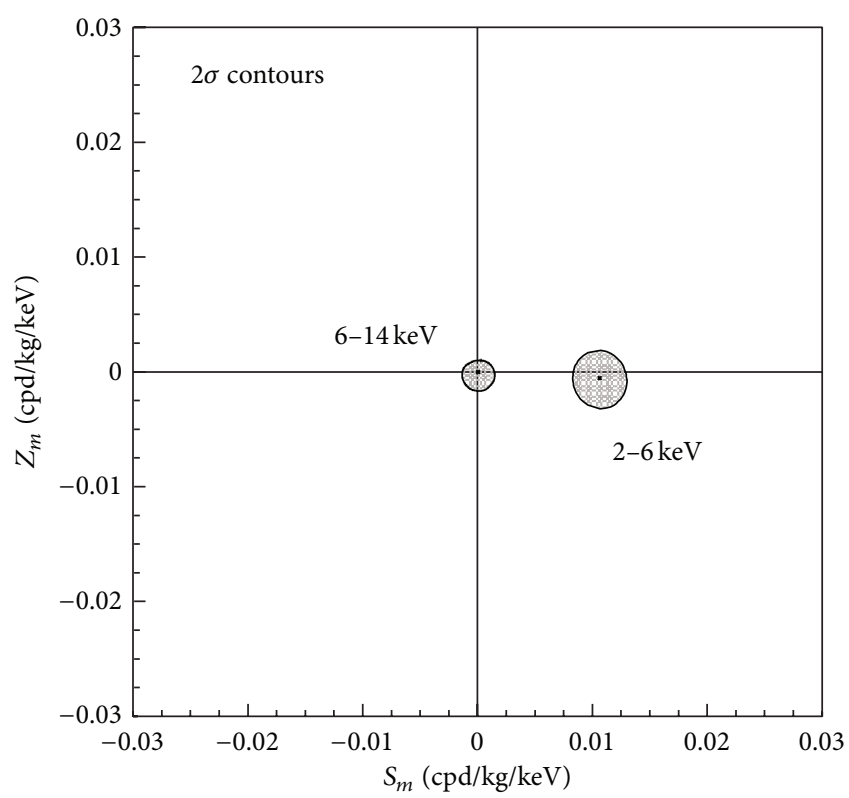

(a)

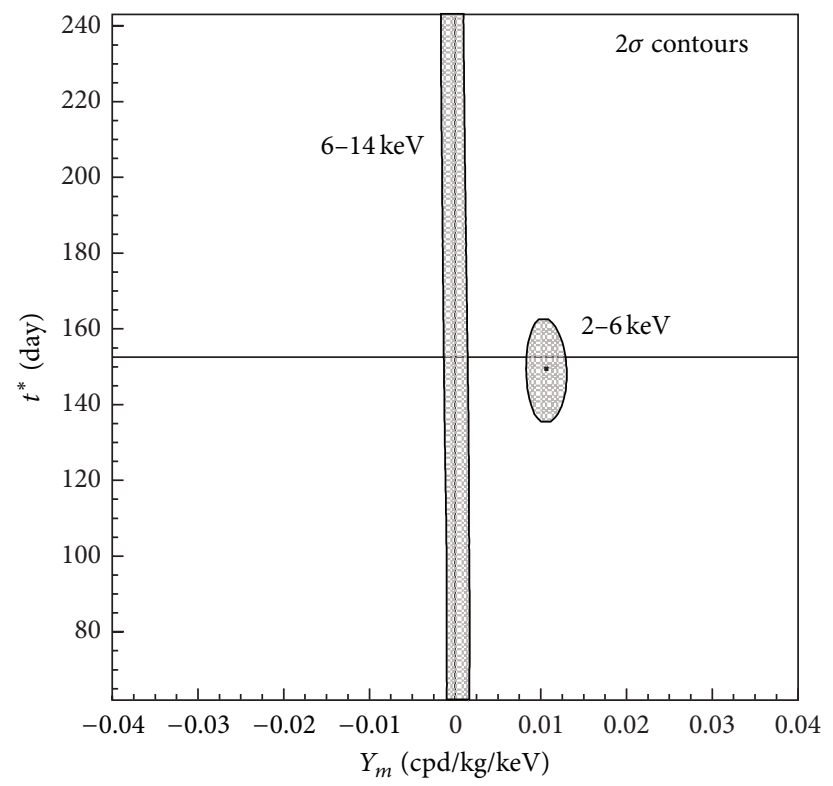

(b)

FIgURE 6: $2 \sigma$ contours in the plane $\left(S_{m}, Z_{m}\right)$ (a) and in the plane $\left(Y_{m}, t^{*}\right)(\mathrm{b})$ for the (2-6) keV and (6-14) keV energy intervals. The contours have been obtained by the maximum likelihood method, considering the cumulative exposure of DAMA/NaI and DAMA/LIBRA-phasel. A modulation amplitude is present in the lower energy intervals and the phase agrees with that expected for DM induced signals. See text.

TABLE 2: Modulation amplitudes ( $1 \sigma$ error) obtained by fitting the time behaviours of the main running parameters including a possible annual modulation with phase and period as for DM particles. These running parameters, acquired with the production data are (i) the operating temperature of the detectors; (ii) the HP Nitrogen flux in the inner Cu box housing the detectors; (iii) the pressure of the HP Nitrogen atmosphere of the inner $\mathrm{Cu}$ box housing the detectors; (iv) the environmental Radon in the inner part of the barrack from which the detectors are, however, excluded; (v) the hardware rate above single photoelectron threshold. All the measured amplitudes are compatible with zero.

\begin{tabular}{lcccc}
\hline & DAMA/LIBRA-1 & DAMA/LIBRA-2 & DAMA/LIBRA-3 & DAMA/LIBRA-4 \\
\hline Temperature $\left({ }^{\circ} \mathrm{C}\right)$ & $-(0.0001 \pm 0.0061)$ & $(0.0026 \pm 0.0086)$ & $(0.001 \pm 0.015)$ & $(0.0004 \pm 0.0047)$ \\
Flux $(\mathrm{L} / \mathrm{h})$ & $(0.13 \pm 0.22)$ & $(0.10 \pm 0.25)$ & $-(0.07 \pm 0.18)$ & $-(0.05 \pm 0.24)$ \\
Pressure $\left(10^{-3} \mathrm{mbar}\right)$ & $(15 \pm 30)$ & $-(13 \pm 25)$ & $(22 \pm 27)$ & $(1.8 \pm 7.4)$ \\
Radon $\left(\mathrm{Bq} / \mathrm{m}^{3}\right)$ & $-(0.029 \pm 0.029)$ & $-(0.030 \pm 0.027)$ & $(0.015 \pm 0.029)$ & $-(0.052 \pm 0.039)$ \\
Hardware rate $\left(10^{-2} \mathrm{~Hz}\right)$ & $-(0.20 \pm 0.18)$ & $(0.09 \pm 0.17)$ & $-(0.03 \pm 0.20)$ & $(0.15 \pm 0.15)$ \\
\hline & DAMA/LIBRA-5 & DAMA/LIBRA-6 & DAMA/LIBRA-7 & $(0.0000 \pm 0.0054)$ \\
Temperature $\left({ }^{\circ} \mathrm{C}\right)$ & $(0.0001 \pm 0.0036)$ & $(0.0007 \pm 0.0059)$ & $-(0.00 \pm 0.14)$ \\
Flux $(\mathrm{L} / \mathrm{h})$ & $-(0.01 \pm 0.21)$ & $-(0.01 \pm 0.15)$ & $-(2.6 \pm 5.5)$ \\
Pressure $\left(10^{-3} \mathrm{mbar}\right)$ & $-(0.8 \pm 1.2)$ & $(0.7 \pm 1.3)$ & $(0.012 \pm 0.047)$ \\
Radon $\left(\mathrm{Bq} / \mathrm{m}^{3}\right)$ & $(0.021 \pm 0.037)$ & $-(0.028 \pm 0.036)$ & $(0.06 \pm 0.10)$ \\
Hardware rate $\left(10^{-2} \mathrm{~Hz}\right)$ & $(0.03 \pm 0.14)$ & $(0.08 \pm 0.11)$ &
\end{tabular}

not able to simultaneously satisfy all the many requirements of the signature. Similar analyses have also been done for the seven annual cycles of DAMA/NaI $[23,24]$.

In conclusion, the model-independent DAMA results give evidence (at 9.3 $\sigma$ C.L. over 14 independent annual cycles) for the presence of DM particles in the galactic halo.

In order to perform corollary investigation on the nature of the DM particles, model-dependent analyses are necessary; thus, many theoretical and experimental parameters and models are possible and many hypotheses must also be exploited.
In particular, the obtained DAMA model independent evidence is compatible with a wide set of scenarios regarding the nature of the DM candidate and related astrophysical, nuclear, and particle Physics. For example, some given scenarios and parameters are discussed in $[2,8,13-20,22,23$, 26]. Further large literature is available on the topics (see e.g., in [8]). Moreover, both the negative results and all the possible positive hints, achieved so far in the field, are largely compatible with the DAMA model-independent DM annual modulation results in many scenarios considering also the existing experimental and theoretical uncertainties; the same 
TABLE 3: Summary of the results obtained by investigating possible sources of systematics or side processes [1-4, 7, 8, 49, 57-62]. None able to give a modulation amplitude different from zero has been found; thus, cautious upper limits (90\% C.L.) on the possible contributions to the measured modulation amplitude have been calculated and are shown here for DAMA/LIBRA-phasel as done before for the seven annual cycles of DAMA/NaI $[23,24]$.

\begin{tabular}{lcc}
\hline Source & Main comment & Cautious upper limit (90\% C.L.) \\
\hline Radon & Sealed Cu Box in HP Nitrogen atmosphere, 3 levels of sealing & $<2.5 \times 10^{-6} \mathrm{cpd} / \mathrm{kg} / \mathrm{keV}$ \\
Temperature & Air conditioning + huge heat capacity & $<10^{-4} \mathrm{cpd} / \mathrm{kg} / \mathrm{keV}$ \\
Noise & Efficient rejection & $<10^{-4} \mathrm{cpd} / \mathrm{kg} / \mathrm{keV}$ \\
Energy scale & Routine + intrinsic calibrations & $<1-2 \times 10^{-4} \mathrm{cpd} / \mathrm{kg} / \mathrm{keV}$ \\
Efficiencies & Regularly measured & $<10^{-4} \mathrm{cpd} / \mathrm{kg} / \mathrm{keV}$ \\
Background & No modulation above $6 \mathrm{keV}$; no modulation in the (2-6) $\mathrm{keV}$ multiple-hit & $<10^{-4} \mathrm{cpd} / \mathrm{kg} / \mathrm{keV}$ \\
Side reactions & events; this limit includes all possible sources of background & $<3 \times 10^{-5} \mathrm{cpd} / \mathrm{kg} / \mathrm{keV}$ \\
\hline
\end{tabular}

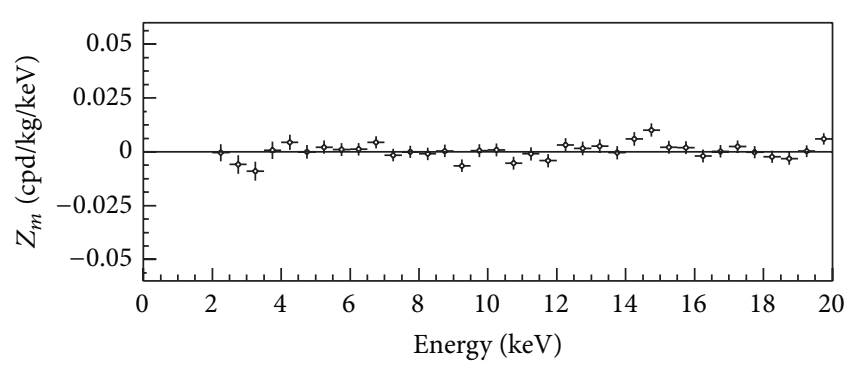

FIGURE 7: $Z_{m}$ variable as a function of the energy for the cumulative exposure of DAMA/NaI and DAMA/LIBRA-phasel, once setting $S_{m}$ in (1) to zero. The energy bin is $0.5 \mathrm{keV}$. The $Z_{m}$ values are expected to be zero. The $\chi^{2}$ test applied to the data supports the hypothesis that the $Z_{m}$ values are simply fluctuating around zero. See text.

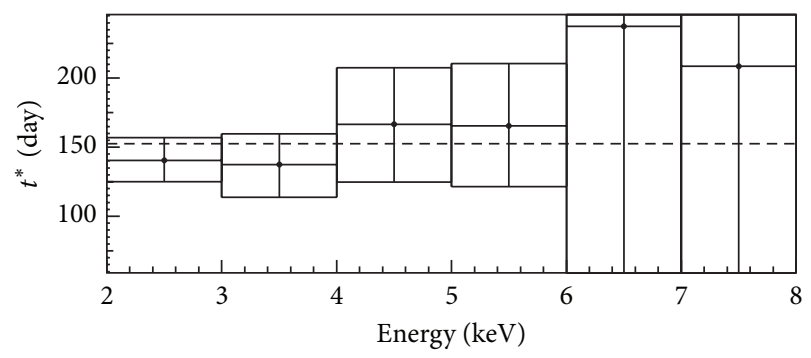

FIGURE 8: Phase $t^{*}$ as a function of the energy for the total exposure; here, the errors are at $2 \sigma$. The vertical scale spans over \pm a quarter of period around 2 June; other intervals are replica of it. An annual modulation effect is present in the lower energy intervals up to $6 \mathrm{keV}$ and the phase agrees with that expected for DM induced signals. No modulation is present above $6 \mathrm{keV}$ and thus the phase is undetermined. See text.

holds for indirect approaches; see, for example, arguments in [8] and references therein. As an example in Figure 9, there are allowed regions shown for DM candidates interacting by elastic scattering on target-nuclei with spin-independent coupling, including also some of the existing uncertainties [5].

\section{DAMA/LIBRA-Phase2 and Perspectives}

A first upgrade of the DAMA/LIBRA setup was performed in September 2008. One detector was recovered by replacing a broken PMT and a new optimization of some PMTs and HVs was done; the transient digitizers were replaced with new ones (the U1063A Acqiris 8-bit 1GS/s DC270 HighSpeed cPCI Digitizers) having better performances and a new DAQ with optical readout was installed. The DAMA/LIBRAphasel concluded its data taking in this configuration on 2010.

A further and more important upgrade has been performed at the end of 2010 when all the PMTs have been replaced with new ones having higher quantum efficiency (Q.E.), realized with a special dedicated development by HAMAMATSU Co. Details on the developments and on the reached performances in the operative conditions are reported in [6]. Just as example, Figure 10 shows the values of the Q.E. of the new 50 HAMAMATSU PMTs installed in DAMA/LIBRA both at peak and at the $\lambda$ of the $\mathrm{NaI}(\mathrm{Tl})$ scintillation light. We remind the reader that up to October 2010 low background PMTs, developed by EMI-Electron Tubes with dedicated R\&D, were used (Q.E. $\simeq 30 \%$ at $\lambda=$ $380 \mathrm{~nm}[1,6,21])$; the light yield and other response features already allowed a software energy threshold of $2 \mathrm{keV}$ in the data analysis. The feasibility to decrease the software energy threshold below $2 \mathrm{keV}$ in the new configuration has been demonstrated [6].

Since the fulfillment of this upgrade, the DAMA/LIBRAphase2 is continuously running in order (1) to increase the experimental sensitivity lowering the software energy threshold of the experiment, (2) to improve the corollary investigation on the nature of the DM particle and related astrophysical, nuclear, and particle physics arguments, and (3) to investigate other signal features. This requires long and heavy full time dedicated work for reliable collection and analysis of very large exposures.

Another upgrade at the end of 2012 was successfully concluded: new-concept preamplifiers were installed, with suitable operative and electronic features; in particular, they allow the direct connection of the signal to the relative channel of the Transient Digitizer (TD). 


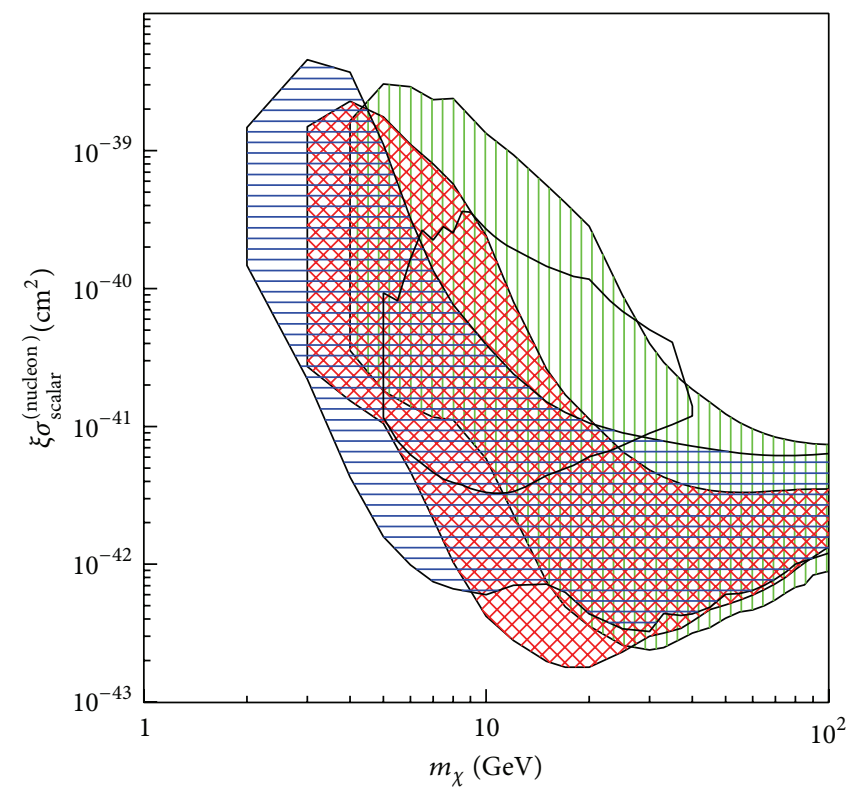

FIGURE 9: Regions in the nucleon cross section versus DM particle mass plane allowed by DAMA for a DM candidate interacting via spinindependent elastic scattering on target-nucleus; three different instances for the $\mathrm{Na}$ and I quenching factors have been considered: (i) without including the channeling effect [(green) vertically hatched region], (ii) by including the channeling effect [(blue) horizontally hatched region)], and (iii) without the channeling effect considering energy dependence of $\mathrm{Na}$ and I quenching factors [5] [(red) crosshatched region]. The velocity distributions and the same uncertainties as in $[23,24]$ are considered here. These regions represent the domain where the likelihoodfunction values differ more than $7.5 \sigma$ from the null hypothesis (absence of modulation). The allowed region obtained for the CoGeNT experiment, including the same astrophysical models as in $[23,24]$ and assuming for simplicity a fixed value for the Ge quenching factor and a Helm form factor with fixed parameters, is also reported by a (black) thick solid line. This region includes configurations whose likelihoodfunction values differ more than $1.64 \sigma$ from the null hypothesis (absence of modulation). For details, see [5].

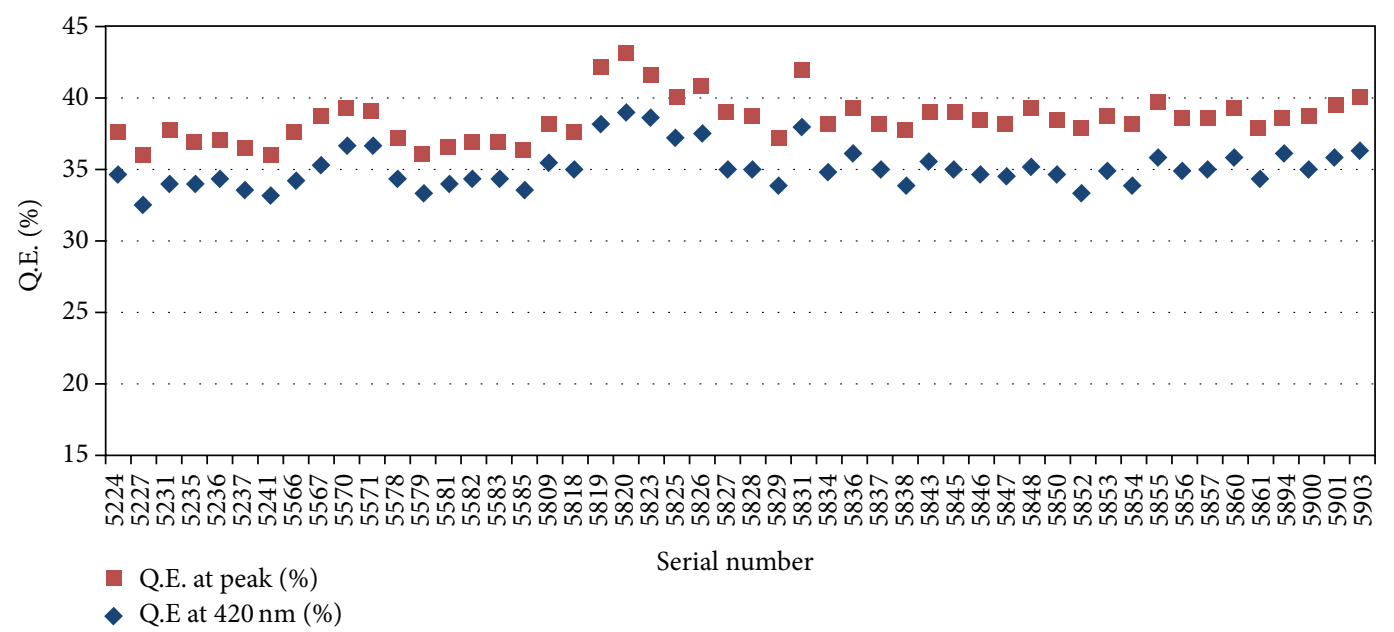

FIgURE 10: Q.E. at peak and at $420 \mathrm{~nm}$ of each one of the 50 high Q.E. PMTs, installed in DAMA/LIBRA-phase2. The averages (RMS) are $38.5 \%$ (1.6\%) and 35.1\% (1.4\%), respectively; the RMS show that the Q.E. spread in the PMTs production is well limited.

Moreover, further improvements are planned. In particular, new trigger modules have been prepared and ready to be installed.

In the future, DAMA/LIBRA will also continue its study on several other rare processes [9-11] as also the former DAMA/NaI apparatus did [31-40].

Finally, further improvements to increase the sensitivity of the setup can be considered; in particular, the use of high
Q.E. and ultra-low background PMTs directly coupled to the $\mathrm{NaI}(\mathrm{Tl})$ crystals is an interesting possibility. (However, this would require the disassembling of the detectors since the light guides act at present also as optical windows.) This possible configuration can allow a further large improvement in the light collection and a further lowering of the software energy threshold. Moreover, efforts towards a possible highly radiopure $\mathrm{NaI}(\mathrm{Tl})$ "general purpose" experiment 
(DAMA/lton) having full sensitive mass of 1 ton (we already proposed in 1996 as a general purpose setup) have been continued in various aspects.

\section{Conclusions}

The data of DAMA/LIBRA-phasel have further confirmed a peculiar annual modulation of the single-hit events in the (2-6) keV energy region satisfying all the many requirements of the DM annual modulation signature; the cumulative exposure by the former DAMA/NaI and DAMA/LIBRAphasel is 1.33 ton $\times y r$.

In fact, as required by the DM annual modulation signature: (1) the single-hit events show a clear cosine-like modulation as expected for the DM signal; (2) the measured period is equal to $(0.998 \pm 0.002) \mathrm{yr}$ well compatible with the $1 \mathrm{yr}$ period as expected for the DM signal; (3) the measured phase, (144 \pm 7 ) days, is compatible with $\simeq 152.5$ days as expected for the DM signal; (4) the modulation is present only in the low energy (2-6) keV interval and not in other higher energy regions, consistently with expectation for the DM signal; (5) the modulation is present only in the single-hit events, while it is absent in the multiple-hit ones as expected for the DM signal; (6) the measured modulation amplitude in $\mathrm{NaI}(\mathrm{Tl})$ of the single-hit events in the (2-6) keV energy interval is $(0.0112 \pm 0.0012) \mathrm{cpd} / \mathrm{kg} / \mathrm{keV}$ (9.3 $\sigma$ C.L.). No systematic or side processes able to simultaneously satisfy all the many peculiarities of the signature and to account for the whole measured modulation amplitude are available.

DAMA/LIBRA is continuously running in its new configuration (named DAMA/LIBRA-phase2) with a lower software energy threshold aiming to improve the knowledge on corollary aspects regarding the signal and on second order effects as discussed, for example, in [8].

\section{Conflict of Interests}

The authors declare that there is no conflict of interests regarding the publication of this paper.

\section{References}

[1] R. Bernabei, P. Belli, A. Bussolotti et al., "The DAMA/LIBRA apparatus," Nuclear Instruments and Methods in Physics Research A, vol. 592, no. 3, pp. 297-315, 2008.

[2] R. Bernabei, P. Belli, F. Cappella et al., "First results from DAMA/LIBRA and the combined results with DAMA/NaI," The European Physical Journal C, vol. 56, no. 3, pp. 333-355, 2008.

[3] R. Bernabei, P. Belli, F. Cappella et al., "New results from DAMA/LIBRA," The European Physical Journal C, vol. 67, no. 1-2, pp. 39-49, 2010.

[4] R. Bernabei, P. Belli, F. Cappella et al., "Final model independent result of DAMA/LIBRA-phasel," The European Physical Journal C, vol. 73, article 2648, 2013.

[5] P. Belli, R. Bernabei, A. Bottino et al., "Observations of annual modulation in direct detection of relic particles and light neutralinos," Physical Review D, vol. 84, no. 5, Article ID 055014, 2011.
[6] R. Bernabei, P. Belli, A. Bussolotti et al., "Performances of the new high quantum efficiency PMTs in DAMA/LIBRA," Journal of Instrumentation, vol. 7, no. 3, Article ID P03009, 2012.

[7] R. Bernabei, P. Belli, F. Cappella et al., "No role for muons in the DAMA annual modulation results," The European Physical Journal C, vol. 72, article 2064, 2012.

[8] R. Bernabei, P. Belli, S. d’Angelo et al., "Dark matter investigation by DAMA at Gran Sasso," International Journal of Modern Physics A, vol. 28, no. 16, Article ID 1330022, 2013.

[9] R. Bernabei, P. Belli, F. Cappella et al., "New search for processes violating the Pauli exclusion principle in sodium and in iodine," The European Physical Journal C, vol. 62, no. 2, pp. 327-332, 2009.

[10] R. Bernabei, P. Belli, F. Cappella et al., "Search for charge nonconserving processes in ${ }^{127} \mathrm{I}$ by coincidence technique," The European Physical Journal C, vol. 72, article 1920, 2012.

[11] R. Bernabei, P. Belli, F. Cappella et al., "New search for correlated $\mathrm{e}^{+} \mathrm{e}^{-}$pairs in the $\alpha$ decay of ${ }^{241}$ Am," The European Physical Journal A, vol. 49, article 64, 2013.

[12] P. Belli, R. Bernabei, C. Bacci, A. Incicchitti, R. Marcovaldi, and D. Prosperi, DAMA proposal to INFN Scientific Committee II, 1990.

[13] R. Bernabei, P. Belli, V. Landoni et al., "New limits on WIMP search with large-mass low-radioactivity $\mathrm{NaI}(\mathrm{Tl})$ set-up at Gran Sasso," Physics Letters B, vol. 389, no. 4, pp. 757-766, 1996.

[14] R. Bernabei, P. Belli, F. Montecchia et al., "Searching for WIMPs by the annual modulation signature," Physics Letters B, vol. 424, no. 1-2, pp. 195-201, 1998.

[15] R. Bernabei, P. Belli, F. Montecchia et al., "On a further search for a yearly modulation of the rate in particle dark matter direct search," Physics Letters B, vol. 450, no. 4, pp. 448-455, 1999.

[16] P. Belli, R. Bernabei, C. J. Dai et al., "Quest for electron decay $e^{-} \rightarrow v_{e} \gamma$ with a liquid xenon scintillator," Physical Review D, vol. 61, no. 11, Article ID 117301, 2000.

[17] R. Bernabei, P. Belli, R. Cerulli et al., "Search for WIMP annual modulation signature: results from DAMA/NaI-3 and DAMA/NaI-4 and the global combined analysis," Physics Letters $B$, vol. 480, no. 1-2, pp. 23-31, 2000.

[18] R. Bernabei, M. Amato, P. Belli et al., "Investigating the DAMA annual modulation data in a mixed coupling framework," Physics Letters B, vol. 509, no. 3-4, pp. 197-203, 2001.

[19] R. Bernabei, P. Belli, R. Cerulli et al., "Investigating the DAMA annual modulation data in the framework of inelastic dark matter," The European Physical Journal C, vol. 23, no. 1, pp. 6164, 2002.

[20] P. Belli, R. Cerulli, N. Fornengo, and S. Scopel, "Effect of the galactic halo modeling on the DAMA-NaI annual modulation result: an extended analysis of the data for weakly interacting massive particles with a purely spin-independent coupling," Physical Review D, vol. 66, no. 4, Article ID 043503, 2002.

[21] R. Bernabei, P. Belli, F. Montecchia et al., "Performances of the $\simeq 100 \mathrm{~kg} \mathrm{NaI}(\mathrm{Tl})$ set-up of the DAMA experiment at Gran Sasso," Il Nuovo Cimento A, vol. 112, no. 6, pp. 545-575, 1999.

[22] R. Bernabei, P. Belli, R. Cerulli et al., "On the investigation of possible systematics in WIMP annual modulation search," The European Physical Journal C, vol. 18, no. 2, pp. 283-292, 2000.

[23] R. Bernabei, P. Belli, F. Cappella et al., "Dark matter search," Rivista del Nuovo Cimento, vol. 26, no. 1, pp. 1-73, 2003.

[24] R. Bernabei, P. Belli, F. Cappella et al., "Dark matter particles in the galactic halo: results and implications from DAMA/NaI," International Journal of Modern Physics D, vol. 13, no. 10, pp. 2127-2159, 2004. 
[25] R. Bernabei, P. Belli, F. Montecchia et al., "Investigating pseudoscalar and scalar dark matter," International Journal of Modern Physics A, vol. 21, no. 7, pp. 1445-1469, 2006.

[26] R. Bernabei, P. Belli, F. Montecchia et al., "Investigating halo substructures with annual modulation signature," The European Physical Journal C, vol. 47, no. 1, pp. 263-271, 2006.

[27] R. Bernabei, P. Belli, F. Montecchia et al., "On electromagnetic contributions in WIMP quests," International Journal of Modern Physics A, vol. 22, no. 19, pp. 3155-3168, 2007.

[28] R. Bernabei, P. Belli, F. Montecchia et al., "Possible implications of the channeling effect in $\mathrm{NaI}(\mathrm{Tl})$ crystals," The European Physical Journal C, vol. 53, no. 2, pp. 205-213, 2008.

[29] R. Bernabei, P. Belli, F. Montecchia et al., "Investigating electron interacting dark matter," Physical Review D, vol. 77, Article ID 023506, 2008.

[30] R. Bernabei, P. Belli, F. Cappella et al., "Investigation on light dark matter," Modern Physics Letters A, vol. 23, no. 26, pp. 21252140, 2008.

[31] R. Bernabei, P. Belli, F. Montecchia et al., "Search for nonpaulian transitions in ${ }^{23} \mathrm{Na}$ and ${ }^{127} \mathrm{I}$," Physics Letters B, vol. 408, no. 1-4, pp. 439-444, 1997.

[32] P. Belli, R. Bernabei, C. J. Dai et al., "New experimental limit on the electron stability and non-paulian transitions in Iodine atoms," Physics Letters B, vol. 460, no. 1-2, pp. 236-241, 1999.

[33] R. Bernabei, P. Belli, R. Cerulli et al., "Extended limits on neutral strongly interacting massive particles and nuclearites from $\mathrm{NaI}(\mathrm{Tl})$ scintillators," Physical Review Letters, vol. 83, no. 24, pp. 4918-4921, 1999.

[34] P. Belli, R. Bernabei, C. J. Dai et al., "New limits on the nuclear levels excitation of ${ }^{127} \mathrm{I}$ and ${ }^{23} \mathrm{Na}$ during charge nonconservation," Physical Review C, vol. 60, no. 6, Article ID 065501, 1999.

[35] R. Bernabei, P. Belli, R. Cerulli et al., "Investigation on possible diurnal effects induced by dark matter particles," Il Nuovo Cimento A, vol. 112, pp. 1541-1552, 1999.

[36] R. Bernabei, P. Belli, R. Cerulli et al., "Search for solar axions by Primakoff effect in NaI crystals," Physics Letters B, vol. 515, no. 1-2, pp. 6-12, 2001.

[37] F. Cappella et al., The European Physical Journal C, vol. 14, p. 1, 2002.

[38] R. Bernabei, P. Belli, F. Cappella et al., "Search for spontaneous transition of nuclei to a superdense state," The European Physical Journal A, vol. 23, no. 1, pp. 7-10, 2005.

[39] R. Bernabei, P. Belli, F. Cappella et al., "A search for spontaneous emission of heavy clusters in the 127I nuclide," The European Physical Journal A, vol. 24, no. 1, pp. 51-56, 2005.

[40] R. Bernabei, P. Belli, W. Di Nicolantonio et al., "A multiton low activity $\mathrm{NaI}(\mathrm{Tl})$ detector for underground physics," Astroparticle Physics, vol. 4, no. 1, pp. 45-54, 1995.

[41] R. Bernabei, The Identification of Dark Matter, World Scientific, Singapore, 1997.

[42] K. A. Drukier, K. Freese, and D. N. Spergel, "Detecting cold dark-matter candidates," Physical Review D, vol. 33, p. 3495, 1986.

[43] K. Freese, J. Frieman, and A. Gould, "Signal modulation in colddark-matter detection," Physical Review D, vol. 37, no. 12, pp. 3388-3405, 1988.

[44] D. Smith and N. Weiner, "Inelastic dark matter," Physical Review D, vol. 64, Article ID 043502, 2001.

[45] D. Tucker-Smith and N. Weiner, "Status of inelastic dark matter," Physical Review D, vol. 72, Article ID 063509, 2005.
[46] D. P. Finkbeiner, T. Lin, and N. Weiner, "Inelastic dark matter and DAMA/LIBRA: an experimentum crucis," Physical Review $D$, vol. 80, Article ID 115008, 2009.

[47] K. Freese, P. Gondolo, and H. J. Newberg, "Detectability of weakly interacting massive particles in the Sagittarius dwarf tidal stream," Physical Review D, vol. 71, Article ID 043516, 2005.

[48] K. Freese, P. Gondolo, H. J. Newberg, and M. Lewis, "Effects of the Sagittarius dwarf tidal stream on dark matter detectors," Physical Review Letters, vol. 92, no. 11, Article ID 11301, 2004.

[49] R. Bernabei, P. Belli, F. Cappella et al., "Technical aspects and dark matter searches," Journal of Physics: Conference Series, vol. 203, no. 1, Article ID 012040, 2010.

[50] K. Freese, P. Gondolo, and H. J. Newberg, "Detectability of weakly interacting massive particles in the Sagittarius dwarf tidal stream," Physical Review D, vol. 71, Article ID 043516, 2005.

[51] P. Gondolo, K. Freese, H. J. Newberg, and M. Lewis, "A dark matter stream through the solar neighborhood," New Astronomy Reviews, vol. 49, no. 2-6, pp. 193-197, 2005.

[52] K. Freese, P. Gondolo, H. J. Newberg, and M. Lewis, "The effects of the Sagittarius dwarf tidal stream on dark matter detectors," Physical Review Letters, vol. 92, Article ID 111301, 2004.

[53] K. Freese, P. Gondolo, and H. J. Newberg, "Detectability of weakly interacting massive particles in the Sagittarius dwarf tidal stream," Physical Review D, vol. 71, Article ID 043516, 2005.

[54] G. Gelmini and P. Gondolo, "Weakly interacting massive particle annual modulation with opposite phase in late-infall halo models," Physical Review D, vol. 64, Article ID 023504, 2001.

[55] F. S. Ling, P. Sikivie, and S. Wick, "Diurnal and annual modulation of cold dark matter signals," Physical Review D, vol. 70, no. 12, Article ID 123503, 2004.

[56] J. Beringer, J.-F. Arguin, R. M. Barnett et al., "Review of particle physics," Physical Review D, vol. 86, Article ID 010001, 2012.

[57] R. Bernabei, P. Belli, F. Cappella et al., "Particle dark matter and DAMA/LIBRA," AIP Conference Proceedings, vol. 1223, p. 50, 2010.

[58] R. Bernabei, P. Belli, F. Cappella et al., "Particle dark matter in DAMA/LIBRA," in Proceedings of the Frontier Objects in Astrophysics and Particle Physics, p. 157, Vulcano, Italy, May 2010, http://arxiv.org/abs/1007.0595.

[59] R. Bernabei, P. Belli, F. Montecchia et al., "Particle dark matter in the galactic halo: recent results from DAMA/LIBRA," Canadian Journal of Physics, vol. 89, no. 1, pp. 141-152, 2011.

[60] R. Bernabei, P. Belli, A. Di Marco et al., "DAMA/LIBRA at Gran Sasso," Physics Procedia, vol. 37, pp. 1095-1104, 2012.

[61] R. Bernabei, P. Belli, F. Cappella et al., "Comment on 'on an unverified nuclear decay and its role in the DAMA experiment,"' http://arxiv.org/abs/1210.6199.

[62] R. Bernabei, P. Belli, F. Cappella et al., "A few final comments to arXiv: 1210.7548 [hep-ph]," http://arxiv.org/abs/1211.6346. 

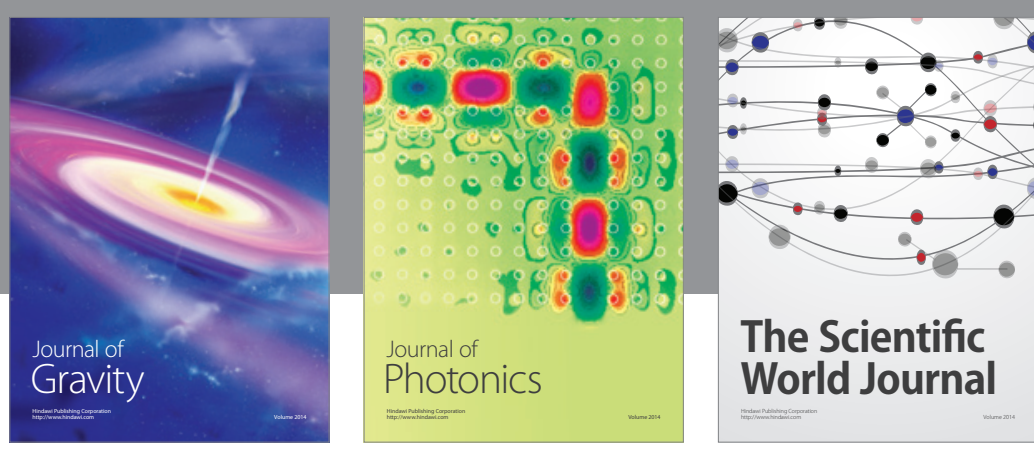

The Scientific World Journal
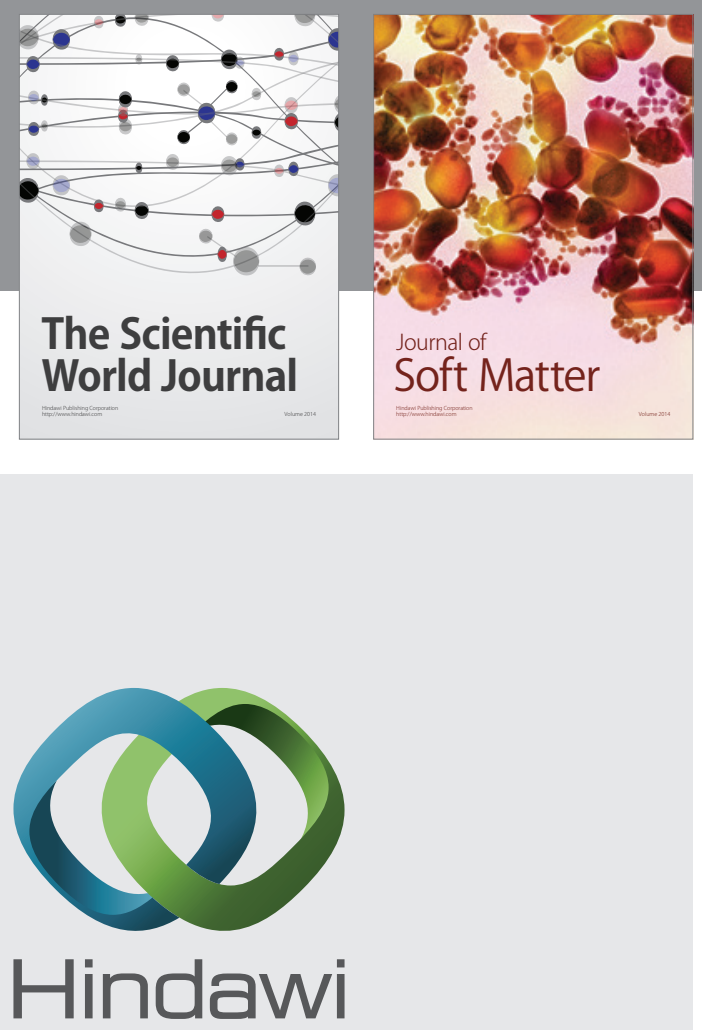

Submit your manuscripts at

http://www.hindawi.com

nternational Journal of

Statistical Mechanics
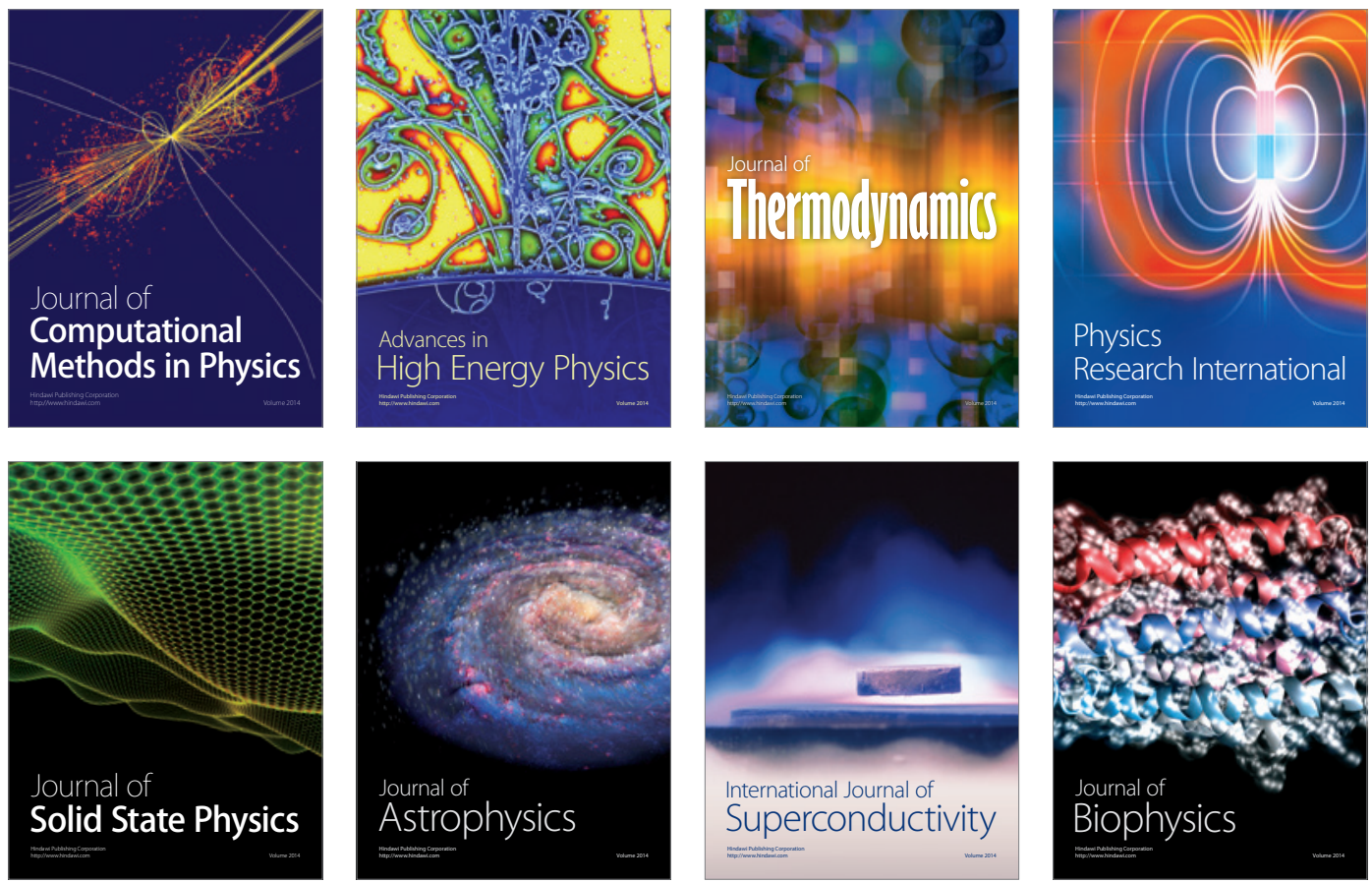
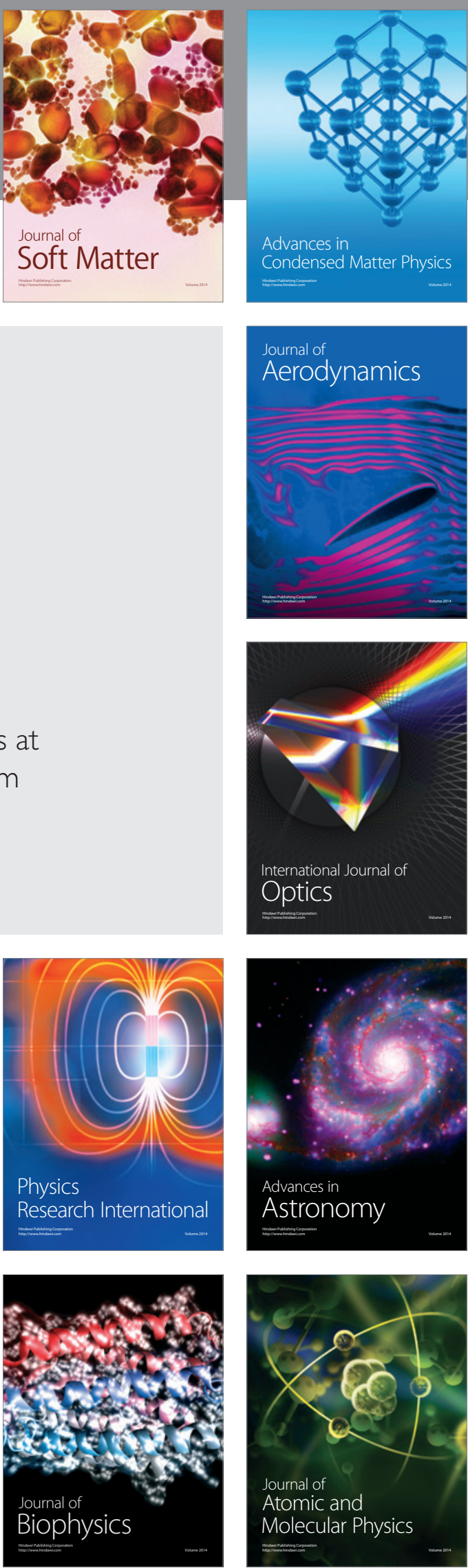